\title{
IMPROVED BECKNER-SOBOLEV INEQUALITIES ON KÄHLER MANIFOLDS
}

\author{
FABRICE BAUDOIN AND OVIDIU MUNTEANU
}

\begin{abstract}
We prove new Beckner-Sobolev type inequalities on compact Kähler manifolds with positive Ricci curvature. As an application, we obtain a diameter upper bound that improves the Bonnet-Myers bound.
\end{abstract}

\section{INTRODUCTION}

Sobolev inequalities have been long studied on Riemannian manifolds. It is well known that on a compact Riemannian manifold $\left(M^{n}, g\right)$ we have a family of inequalities of the type

$$
\left(\int_{M}|\phi|^{p}\right)^{\frac{2}{p}} \leq A \int_{M}|\nabla \phi|^{2}+B \int_{M} \phi^{2}
$$

for any $\phi \in C^{\infty}(M)$ and $\frac{2 n}{n-2} \geq p>2$, where $A$ and $B$ are some fixed constants that may depend on $M$.

It is an important question to obtain sharp bounds for the constants $A$ or $B$. An important result of Hebey-Vaugon [11 says that for $p=\frac{2 n}{n-2}$ the constant $A$ may be taken to be the optimal constant in the Sobolev inequality on $\mathbb{R}^{n}$, that is $A=\frac{4}{n(n-2) \omega_{n}^{2 / n}}$, where $\omega_{n}$ is the volume of the unit sphere in $\mathbb{R}^{n+1}$. We refer to 10] for an excellent reference on the $A B$ program.

Our interest lies in the case when $B=1$, that is

$$
\left(\int_{M}|\phi|^{p}\right)^{\frac{2}{p}}-\int_{M} \phi^{2} \leq A \int_{M}|\nabla \phi|^{2}
$$

for any $\phi \in C^{\infty}(M)$ and $\frac{2 n}{n-2} \geq p>2$. Here and below we normalized the volume so that $\operatorname{Vol}(M)=1$. The best constant $A$ can be estimated geometrically in terms of a Ricci curvature lower bound on the manifold.

Theorem 1. Let $\left(M^{n}, g\right)$ be a compact Riemannian manifold with Ricci curvature Ric $\geq \rho$, for some constant $\rho>0$. Then

$$
\left(\int_{M}|\phi|^{p}\right)^{\frac{2}{p}}-\int_{M} \phi^{2} \leq \frac{(n-1)(p-2)}{n \rho} \int_{M}|\nabla \phi|^{2}
$$

for all $\phi \in C^{\infty}(M)$.

This was proved by Bidaut-Veron and Veron in [5], using a method proposed in 9]. In this form, the inequality actually holds for $1 \leq p \leq 2$ as well, which are

The first author was partially supported by NSF grant DMS-1660031 and the Simons Foundation. The second author was partially supported by NSF grant DMS- 1506220 . 
called Beckner inequalities [8]. These interpolate between the Poincaré inequality for $p=1$ and the $\log$ Sobolev inequality for $p \rightarrow 2$.

On the class of Riemannian manifolds with Ric $\geq \rho$, the constant $\frac{(n-1)(p-2)}{n \rho}$ in (1.1) can not be improved since it is optimal for the spheres, see for instance [7. Our goal in this paper is to improve the constant $\frac{(n-1)(p-2)}{n \rho}$ in Theorem 1 in the Kähler setting. We have been able to do so for the entire range $1 \leq p<\frac{2 n}{n-2}$, by using an improved integrated Bochner's inequality that takes into account the Kähler structure (see Theorem 44). The following is the main result of this paper.

Theorem 2. Let $\left(M^{m}, g\right)$ be a compact Kähler manifold of complex dimension $m \geq 2$ and with Ricci curvature $\operatorname{Ric} \geq \rho$ with $\rho>0$ and $\operatorname{Vol}(M)=1$. For any $2<p \leq \frac{2 m}{m-1}$ we have the Sobolev inequality

$$
\left(\int_{M}|\phi|^{p}\right)^{\frac{2}{p}}-\int_{M} \phi^{2} \leq C_{S} \int_{M}|\nabla \phi|^{2}
$$

for any $\phi \in C^{\infty}(M)$, where

$$
C_{S}=\frac{p-2}{(p-1) 2 m \rho}(2 m+p+1-2 \sqrt{(m+1)(2 m-(m-1) p)}) .
$$

For any $1<p \leq 2$ we have the Beckner inequality

$$
\int_{M} \phi^{2}-\left(\int_{M} \phi^{\frac{2}{p}}\right)^{p} \leq C_{B} \int_{M}|\nabla \phi|^{2},
$$

for any $\phi \in C^{\infty}(M)$, where

$$
C_{B}=\frac{p-1}{p} \frac{2 m}{((m-1) p+2) \rho} .
$$

Let us note that Sobolev inequality on Kähler manifolds improves that in Theorem 1 for all $1 \leq p<\frac{2 n}{n-2}=\frac{2 m}{m-1}$. In the critical case $p=\frac{2 n}{n-2}$ the optimal Sobolev constant is related to the Yamabe invariant, and so it cannot be improved in the Kähler setting. Indeed, by Obata theorem [16, any non-spherical Einstein metric is the unique constant scalar curvature metric in its conformal class, which implies that Kähler Einstein metrics have the same Sobolev constants as spheres.

The Beckner inequalities also refine those from Theorem [1. In particular, letting $p \rightarrow 1$, we have a log-Sobolev inequality of the form

$$
\int_{M} \phi^{2} \ln \phi^{2}-\int_{M} \phi^{2} \ln \int_{M} \phi^{2} \leq \frac{2 m}{(m+1) \rho} \int_{M}|\nabla \phi|^{2},
$$

and for $p=2$ a sharp Poincaré inequality of the form

$$
\int_{M} \phi^{2}-\left(\int_{M} \phi\right)^{2} \leq \frac{1}{2 \rho} \int_{M}|\nabla \phi|^{2}
$$

It is not clear to us at this point if the Beckner-Sobolev constants we obtain are optimal for other values of $p$ than 2. Let us however point out that according to Theorem 1.3 in [17, for the $\log$-Sobolev inequality $p=1$, the constant $\frac{2 m}{(m+1) \rho}$ is asymptotically sharp in the case of the complex projective space $\mathbb{C P}^{m}$ when $m \rightarrow+\infty$.

As an application of these results, we use a theory developed by Bakry and Ledoux [3] to estimate the diameter of such manifolds. The Bonnet-Myers diameter estimate asserts that a complete $n$-dimensional Riemannian manifold $\left(M^{n}, g\right)$ with 
Ricci curvature lower bound Ric $\geq n-1$ is compact and has a sharp diameter upper bound $\operatorname{diam}(M) \leq \pi$. By Cheng's theorem, equality is achieved if $M$ is a sphere of constant sectional curvature. Since the rigidity in Bonnet-Myers theorem does not apply to Kähler manifolds for complex dimension $m \geq 2$, it is a natural question to study the diameter bound in this setting. Several results are known. If $\left(M^{m}, g\right)$ has bisectional curvature bounded by $B K \geq 1$, it is known [13] that $\operatorname{diam}(M) \leq \frac{\pi}{2}$, this result is sharp and equality is achieved by the complex projective space $\mathbb{C P}^{m}$. Rigidity in this estimate has been studied recently in [19, 15]. In fact, it should be noted that this sharp estimate holds under the weaker lower bound on holomorphic sectional curvature, [18.

On the other hand, one cannot replace the bisectional curvature by Ricci curvature in the Kähler case, because the Kähler-Einstein metric on $\mathbb{C P}^{1} \times \mathbb{C P}^{1} \times . . \times \mathbb{C P}^{1}$ has larger diameter than $\mathbb{C P}^{m}$. A sharp diameter estimate for Kähler manifolds $\left(M^{m}, g\right)$ with Ric $\geq 2 m-1$ is currently not known, but Liu proved [14] that there exists a constant $\varepsilon(m)$ depending only on $m$ so that $\operatorname{diam}(M) \leq \pi-\varepsilon(m)$, for any Kähler manifold satisfying Ric $\geq 2 m-1$. However, the precise dependency of $\varepsilon$ on the dimension $m$ in 14 is somewhat difficult to state.

As an application of Theorem 2 we obtain the following.

Theorem 3. Let $\left(M^{m}, g\right)$ be a Kähler manifold of complex dimension $m \geq 2$ and with Ricci curvature Ric $\geq 2 m-1$. Then

$$
\operatorname{diam}(M) \leq \pi\left(1-\frac{1}{24 m}\right)
$$

The organization of the paper is as follows. In Section 2 we present a new Bochner type argument for Kähler manifolds. This is used in Section 3 to prove Theorem 2 Finally, the diameter estimate is proved in Section 4.

Acknowledgements. It is our pleasure to thank Xiaodong Wang and Jiaping Wang for useful discussions and for their interest in this work.

\section{A Differential inequality for KäHLER MANifoldS}

Let us set the notation that will be used throughout this note. Let $\left(M^{m}, g\right)$ be a compact Kähler manifold of complex dimension $m$. The Kähler metric $d s^{2}=$ $g_{\alpha \bar{\beta}} d z^{\alpha} d \bar{z}^{\beta}$ defines a Riemannian metric by $\operatorname{Re}\left(d s^{2}\right)$. Hence, if $\left\{e_{k}\right\}_{k=1,2 m}$ is an orthonormal frame so that $e_{2 k}=J e_{2 k-1}$, then $\left\{\nu_{\alpha}\right\}_{\alpha=1, m}$

$$
\nu_{\alpha}=\frac{1}{2}\left(e_{2 \alpha-1}-\sqrt{-1} e_{2 \alpha}\right)
$$

is a unitary frame. It follows that in this normalization of the Riemannian metric, we have

$$
\begin{aligned}
\Delta f & =4 f_{\alpha \bar{\alpha}} \\
\langle\nabla f, \nabla h\rangle & =2\left(f_{\alpha} h_{\bar{\alpha}}+f_{\bar{\alpha}} h_{\alpha}\right) .
\end{aligned}
$$

A lower bound for Ricci curvature Ric $\geq \rho$ means that $\operatorname{Ric}\left(e_{j}, e_{k}\right) \geq \rho \delta_{j k}$, or in the unitary frame $\left\{\nu_{\alpha}\right\}_{\alpha=1, m}$ that $R_{\alpha \bar{\beta}} \geq \frac{1}{2} \rho \delta_{\alpha \bar{\beta}}$. 
Let $a \in \mathbb{R}$ and $k, q \geq 0$ be arbitrary. We fix the following constants

$$
\begin{aligned}
A_{1}= & 2(m+1) a k-(1+2 k) m q \\
B_{1}= & ((m-1)+m k) k a^{2}+(2-(k+1) q) m k a \\
& +\frac{m}{4}(1-k)^{2} q^{2}+k m q(q-1),
\end{aligned}
$$

and

$$
\begin{aligned}
A_{2}= & 2(m+1) a k+\frac{1}{2}(1-k) m q-\frac{3}{2} k q \\
B_{2}= & ((m-1)+m k) k a^{2}+(2-(k+1) q) m k a \\
& +\frac{m}{4}(1-k)^{2} q^{2}+\frac{q(q-1)}{2}(m(k-1)+k) .
\end{aligned}
$$

We have the following integral estimates which are the keys to our results and improve upon the integrated curvature dimension type inequality available on arbitrary Riemannian manifolds.

Theorem 4. Let $\left(M^{m}, g\right)$ be a compact Kähler manifold of complex dimension $m \geq 2$ and with Ricci curvature Ric $\geq \rho$ for some $\rho>0$. For any function $u>0$, any $a \in \mathbb{R}$ and $k, q \geq 0$, we have

$$
\begin{aligned}
(m+(m-1) k) \int_{M}(\Delta u)^{2} u^{q} \geq & 2 m \rho \int_{M}|\nabla u|^{2} u^{q}+A_{1} \int_{M}|\nabla u|^{2}(\Delta u) u^{q-1} \\
& -B_{1} \int_{M}|\nabla u|^{4} u^{q-2},
\end{aligned}
$$

where $A_{1}$ and $B_{1}$ are specified in (2.1). If $0 \leq k \leq \frac{m}{m-1}$, then for any $u>0$ and any $q \geq 0$ we have

$$
\begin{aligned}
(m+(m-1) k) \int_{M}\left|\nabla^{2} u\right|^{2} u^{q} \geq & (m-(m-1) k) \rho \int_{M}|\nabla u|^{2} u^{q}+A_{2} \int_{M}|\nabla u|^{2}(\Delta u) u^{q-1} \\
& -B_{2} \int_{M}|\nabla u|^{4} u^{q-2},
\end{aligned}
$$

for $A_{2}$ and $B_{2}$ specified in (2.2).

Proof. It suffices to prove the theorem for any $k, q>0$, and any $a \in \mathbb{R}$. We have by the arithmetic-mean inequality that

$$
\left|u_{\alpha \bar{\beta}}+a \frac{u_{\alpha} u_{\bar{\beta}}}{u}\right|^{2} \geq \frac{1}{16 m}\left|\Delta u+a \frac{|\nabla u|^{2}}{u}\right|^{2} .
$$

Expanding the terms, this implies

$$
\begin{aligned}
& \left|u_{\alpha \bar{\beta}}\right|^{2}+2 a \frac{u_{\alpha \bar{\beta}} u_{\bar{\alpha}} u_{\beta}}{u}+\frac{a^{2}}{16} \frac{m-1}{m} \frac{|\nabla u|^{4}}{u^{2}} \\
\geq & \frac{1}{16 m}(\Delta u)^{2}+\frac{a}{8 m} \frac{|\nabla u|^{2}}{u} \Delta u .
\end{aligned}
$$


Multiplying this with $u^{q}$ it follows that

$$
\begin{aligned}
\int_{M}\left|u_{\alpha \bar{\beta}}\right|^{2} u^{q} \geq & \frac{1}{16 m} \int_{M}(\Delta u)^{2} u^{q}+\frac{a}{8 m} \int_{M}|\nabla u|^{2}(\Delta u) u^{q-1} \\
& -2 a \int_{M}\left(u_{\alpha \bar{\beta}} u_{\bar{\alpha}} u_{\beta}\right) u^{q-1} \\
& -\frac{a^{2}}{16} \frac{m-1}{m} \int_{M}|\nabla u|^{4} u^{q-2} .
\end{aligned}
$$

For $b \in \mathbb{R}$ to be specified later we note the following inequality

$$
\left|u_{\alpha \beta}\right|^{2}+2 b \frac{\operatorname{Re}\left(u_{\alpha \beta} u_{\bar{\alpha}} u_{\bar{\beta}}\right)}{u}+\frac{b^{2}}{16} \frac{|\nabla u|^{4}}{u^{2}} \geq 0,
$$

where $\operatorname{Re}(w)=\frac{1}{2}(w+\bar{w})$ denotes the real part of $w$. We get that

$$
\int_{M}\left|u_{\alpha \beta}\right|^{2} u^{q} \geq-2 b \int_{M} \operatorname{Re}\left(u_{\alpha \beta} u_{\bar{\alpha}} u_{\bar{\beta}}\right) u^{q-1}-\frac{b^{2}}{16} \int_{M}|\nabla u|^{4} u^{q-2} .
$$

Integrating by parts, we obtain the following

$$
\begin{aligned}
-\int_{M}\left(u_{\alpha \beta} u_{\bar{\alpha}} u_{\bar{\beta}}\right) u^{q-1}= & \int_{M} u_{\alpha}\left(\left(u_{\bar{\alpha}} u_{\bar{\beta}}\right) u^{q-1}\right)_{\beta} \\
= & \int_{M}\left(u_{\bar{\alpha} \beta} u_{\alpha} u_{\bar{\beta}}\right) u^{q-1}+\frac{1}{16} \int_{M}|\nabla u|^{2}(\Delta u) u^{q-1} \\
& +\frac{q-1}{16} \int_{M}|\nabla u|^{4} u^{q-2}
\end{aligned}
$$

Hence, we have

$$
\begin{aligned}
-\int_{M} \operatorname{Re}\left(u_{\alpha \beta} u_{\bar{\alpha}} u_{\bar{\beta}}\right) u^{q-1}= & \int_{M}\left(u_{\bar{\alpha} \beta} u_{\alpha} u_{\bar{\beta}}\right) u^{q-1} \\
& +\frac{1}{16} \int_{M}|\nabla u|^{2}(\Delta u) u^{q-1} \\
& +\frac{q-1}{16} \int_{M}|\nabla u|^{4} u^{q-2} .
\end{aligned}
$$

Using this in (2.4) proves that

$$
\begin{aligned}
\int_{M}\left|u_{\alpha \beta}\right|^{2} u^{q} \geq & 2 b \int_{M}\left(u_{\bar{\alpha} \beta} u_{\alpha} u_{\bar{\beta}}\right) u^{q-1}+\frac{b}{8} \int_{M}|\nabla u|^{2}(\Delta u) u^{q-1} \\
& -\left(\frac{b^{2}}{16}+\frac{b(1-q)}{8}\right) \int_{M}|\nabla u|^{4} u^{q-2} .
\end{aligned}
$$

Multiply (2.6) by $\frac{1}{k}>0$ and add to (2.3) to conclude

$$
\begin{aligned}
& \int_{M}\left|u_{\alpha \bar{\beta}}\right|^{2} u^{q}+\frac{1}{k} \int_{M}\left|u_{\alpha \beta}\right|^{2} u^{q} \\
\geq & \frac{1}{16 m} \int_{M}(\Delta u)^{2} u^{q}+\left(\frac{a}{8 m}+\frac{b}{8 k}\right) \int_{M}|\nabla u|^{2}(\Delta u) u^{q-1} \\
& -2\left(a-\frac{b}{k}\right) \int_{M}\left(u_{\alpha \bar{\beta}} u_{\bar{\alpha}} u_{\beta}\right) u^{q-1} \\
& -\left(\frac{m-1}{16 m} a^{2}+\frac{b^{2}}{16 k}+\frac{b(1-q)}{8 k}\right) \int_{M}|\nabla u|^{4} u^{q-2} .
\end{aligned}
$$


We now integrate by parts and use Ricci identities to get

$$
\begin{aligned}
\int_{M}\left|u_{\alpha \bar{\beta}}\right|^{2} u^{q}= & -\int_{M}\left(u_{\alpha \bar{\beta} \beta} u_{\bar{\alpha}}\right) u^{q}-q \int_{M}\left(u_{\alpha \bar{\beta}} u_{\bar{\alpha}} u_{\beta}\right) u^{q-1} \\
= & -\frac{1}{16} \int_{M}\langle\nabla \Delta u, \nabla u\rangle u^{q}-q \int_{M}\left(u_{\alpha \bar{\beta}} u_{\bar{\alpha}} u_{\beta}\right) u^{q-1} \\
= & \frac{1}{16} \int_{M}(\Delta u)^{2} u^{q}+\frac{q}{16} \int_{M}|\nabla u|^{2}(\Delta u) u^{q-1} \\
& -q \int_{M}\left(u_{\alpha \bar{\beta}} u_{\bar{\alpha}} u_{\beta}\right) u^{q-1} .
\end{aligned}
$$

From (2.8) we get

$$
\begin{aligned}
\int_{M}\left(u_{\alpha \bar{\beta}} u_{\bar{\alpha}} u_{\beta}\right) u^{q-1}= & -\frac{1}{q} \int_{M}\left|u_{\alpha \bar{\beta}}\right|^{2} u^{q}+\frac{1}{16 q} \int_{M}(\Delta u)^{2} u^{q} \\
& +\frac{1}{16} \int_{M}|\nabla u|^{2}(\Delta u) u^{q-1},
\end{aligned}
$$

that we plug into (2.7) to obtain

$$
\begin{aligned}
& \int_{M}\left(1-\frac{2}{q}\left(a-\frac{b}{k}\right)\right)\left|u_{\alpha \bar{\beta}}\right|^{2} u^{q}+\frac{1}{k} \int_{M}\left|u_{\alpha \beta}\right|^{2} u^{q} \\
\geq & \left(\frac{1}{16 m}-\frac{1}{8 q}\left(a-\frac{b}{k}\right)\right) \int_{M}(\Delta u)^{2} u^{q} \\
& +\left(-\frac{m-1}{8 m} a+\frac{b}{4 k}\right) \int_{M}|\nabla u|^{2}(\Delta u) u^{q-1} \\
& -\left(\frac{m-1}{16 m} a^{2}+\frac{b^{2}}{16 k}+\frac{b(1-q)}{8 k}\right) \int_{M}|\nabla u|^{4} u^{q-2} .
\end{aligned}
$$

We now set

$$
b=k a+(1-k) \frac{q}{2}
$$

for which

$$
1-\frac{2}{q}\left(a-\frac{b}{k}\right)=\frac{1}{k}
$$

Noting that

$$
\left|\nabla^{2} u\right|^{2}=8\left(\left|u_{\alpha \bar{\beta}}\right|^{2}+\left|u_{\alpha \beta}\right|^{2}\right)
$$

we get from (2.9) that

$$
\begin{aligned}
\int_{M}\left|\nabla^{2} u\right|^{2} u^{q} \geq & \frac{m-(m-1) k}{2 m} \int_{M}(\Delta u)^{2} u^{q} \\
& +A \int_{M}|\nabla u|^{2}(\Delta u) u^{q-1} \\
& -B \int_{M}|\nabla u|^{4} u^{q-2}
\end{aligned}
$$


where

$$
\begin{aligned}
A= & \frac{m+1}{m} a k+(1-k) q \\
B= & \frac{1}{2}\left(\frac{m-1}{m}+k\right) k a^{2}+\left(\frac{q}{2}(1-k)+(1-q)\right) k a \\
& +\frac{1}{8}(1-k)^{2} q^{2}+\frac{1}{2}(1-k) q(1-q) .
\end{aligned}
$$

We now use the Ricci curvature lower bound by integrating $\int_{M}\left|\nabla^{2} u\right|^{2} u^{q}$ by parts. From

$$
\frac{1}{2} \Delta|\nabla u|^{2}=\left|\nabla^{2} u\right|^{2}+\langle\nabla \Delta u, \nabla u\rangle+\operatorname{Ric}(\nabla u, \nabla u)
$$

we obtain

$$
\begin{aligned}
\int_{M}\left|\nabla^{2} u\right|^{2} u^{q} \leq & -\int_{M}\langle\nabla \Delta u, \nabla u\rangle u^{q}+\frac{1}{2} \int_{M}\left(\Delta|\nabla u|^{2}\right) u^{q} \\
& -\rho \int_{M}|\nabla u|^{2} u^{q} \\
= & \int_{M}(\Delta u)^{2} u^{q}+q \int_{M}|\nabla u|^{2}(\Delta u) u^{q-1} \\
& -\frac{q}{2} \int_{M}\left\langle\nabla|\nabla u|^{2}, \nabla u\right\rangle u^{q-1}-\rho \int_{M}|\nabla u|^{2} u^{q} \\
= & \int_{M}(\Delta u)^{2} u^{q}+\frac{3 q}{2} \int_{M}|\nabla u|^{2}(\Delta u) u^{q-1} \\
& +\frac{q(q-1)}{2} \int_{M}|\nabla u|^{4} u^{q-2}-\rho \int_{M}|\nabla u|^{2} u^{q} .
\end{aligned}
$$

This proves that

$$
\begin{aligned}
\int_{M}(\Delta u)^{2} u^{q} \geq & \int_{M}\left|\nabla^{2} u\right|^{2} u^{q}-\frac{3 q}{2} \int_{M}|\nabla u|^{2}(\Delta u) u^{q-1} \\
& -\frac{q(q-1)}{2} \int_{M}|\nabla u|^{4} u^{q-2}+\rho \int_{M}|\nabla u|^{2} u^{q} .
\end{aligned}
$$

Plugging (2.10) into (2.12) we conclude that

$$
\begin{aligned}
(m+(m-1) k) \int_{M}(\Delta u)^{2} u^{q} \geq & 2 m \rho \int_{M}|\nabla u|^{2} u^{q} \\
& +A_{1} \int_{M}|\nabla u|^{2}(\Delta u) u^{q-1} \\
& -B_{1} \int_{M}|\nabla u|^{4} u^{q-2}
\end{aligned}
$$

where

$$
\begin{aligned}
A_{1}= & 2(m+1) a k-(1+2 k) m q \\
B_{1}= & ((m-1)+m k) k a^{2}+(2-(k+1) q) m k a \\
& +\frac{1}{4}(1-k)^{2} m q^{2}+m k q(q-1) .
\end{aligned}
$$

We now assume that

$$
k \leq \frac{m}{m-1}
$$


Plugging (2.13) into (2.10) we now get

$$
\begin{aligned}
(m+(m-1) k) \int_{M}\left|\nabla^{2} u\right|^{2} u^{q} \geq & (m-(m-1) k) \rho \int_{M}|\nabla u|^{2} u^{q} \\
& +A_{2} \int_{M}|\nabla u|^{2}(\Delta u) u^{q-1} \\
& -B_{2} \int_{M}|\nabla u|^{4} u^{q-2},
\end{aligned}
$$

where

$$
\begin{aligned}
A_{2}= & 2(m+1) a k+\frac{1}{2}(1-k) m q-\frac{3}{2} k q \\
B_{2}= & ((m-1)+m k) k a^{2}+(2-(k+1) q) m k a \\
& +\frac{m}{4}(1-k)^{2} q^{2}+\frac{q(q-1)}{2}(m(k-1)+k) .
\end{aligned}
$$

By (2.13) and (2.15) we obtain the result.

Let us note that for $k=1$ and $q=0,(2.13)$ recovers the Bochner inequality on p. 338 of [12], and for $k=1$ (2.15) recovers the Bochner inequality on p.13 of [8].

\section{Beckner-Sobolev inequalities}

Recall that on a Riemannian manifold $\left(M^{n}, g\right)$ with Ricci curvature bounded below by Ric $\geq \rho$ and volume normalized by $\operatorname{Vol}(M)=1$, the following sharp Sobolev inequality holds [12, 5]

$$
\left(\int_{M}|\phi|^{p}\right)^{\frac{2}{p}}-\int_{M} \phi^{2} \leq \frac{(n-1)(p-2)}{n \rho} \int_{M}|\nabla \phi|^{2},
$$

for any $\phi \in C^{\infty}(M)$ and $2<p \leq \frac{2 n}{n-2}$. Our goal is to improve (3.1) in the Kähler setting. As a preparation, we prove the following result.

Lemma 5. Let $\left(M^{m}, g\right)$ be a compact Kähler manifold satisfying Ric $\geq \rho$ with $\rho>0$. Assume there exists a smooth nonconstant positive solution $f$ of

$$
\Delta f=C\left(f-f^{p-1}\right),
$$

for some $2<p \leq \frac{2 m}{m-1}$ and some constant $C \in \mathbb{R}$. Then

$$
C \geq \frac{2 m \rho}{(m+(m-1) k)(p-2)},
$$

for any $k>0$ such that

$$
p \leq 1+\frac{m+1}{m-1} \frac{4 k}{(k+1)^{2}}
$$

Proof. We follow the approach in [5], see also [4, 12] and Ch.6.8 of [2]. Define

$$
u=f^{\frac{1}{r}}
$$

for some $r \in \mathbb{R}$ to be determined later. 
We claim the following identity

$$
\begin{aligned}
C(p-2) \int_{M}|\nabla u|^{2}= & \int_{M}(\Delta u)^{2}+r(p-1) \int_{M} \frac{|\nabla u|^{2}}{u} \Delta u \\
& +(r-1)(r(p-2)+1) \int_{M} \frac{|\nabla u|^{4}}{u^{2}} .
\end{aligned}
$$

Indeed, since $f=u^{r}$ and $\Delta f=C\left(f-f^{p-1}\right)$, we get the following equation for $u$

$$
r u^{r-1} \Delta u+r(r-1) u^{r-2}|\nabla u|^{2}=C u^{r}-C u^{r(p-1)} .
$$

Multiply (3.3) by $|\nabla u|^{2} u^{-r}$ to get

$$
r \int_{M} \frac{|\nabla u|^{2}}{u} \Delta u+r(r-1) \int_{M} \frac{|\nabla u|^{4}}{u^{2}}=C \int_{M}|\nabla u|^{2}-C \int_{M} u^{r(p-2)}|\nabla u|^{2} .
$$

We can write using integration by parts

$$
\begin{aligned}
\int_{M} u^{r(p-2)}|\nabla u|^{2} & =\frac{1}{r(p-2)+1} \int_{M}\left\langle\nabla u, \nabla u^{r(p-2)+1}\right\rangle \\
& =-\frac{1}{r(p-2)+1} \int_{M} u^{r(p-2)+1} \Delta u .
\end{aligned}
$$

Hence, (3.4) becomes

$$
\begin{aligned}
& r \int_{M} \frac{|\nabla u|^{2}}{u} \Delta u+r(r-1) \int_{M} \frac{|\nabla u|^{4}}{u^{2}} \\
= & C \int_{M}|\nabla u|^{2}+\frac{C}{r(p-2)+1} \int_{M} u^{r(p-2)+1} \Delta u .
\end{aligned}
$$

To compute the second term on the right side of (3.5) we multiply (3.3) by $u^{-r+1} \Delta u$ and get

$$
r \int_{M}(\Delta u)^{2}+r(r-1) \int_{M} \frac{|\nabla u|^{2}}{u} \Delta u=-C \int_{M}|\nabla u|^{2}-C \int_{M} u^{r(p-2)+1} \Delta u .
$$

This proves that

$$
\begin{aligned}
\frac{C}{r(p-2)+1} \int_{M} u^{r(p-2)+1} \Delta u= & -\frac{r}{r(p-2)+1} \int_{M}(\Delta u)^{2}-\frac{r(r-1)}{r(p-2)+1} \int_{M} \frac{|\nabla u|^{2}}{u} \Delta u \\
& -\frac{C}{r(p-2)+1} \int_{M}|\nabla u|^{2} .
\end{aligned}
$$

Plugging this in (3.5) implies that

$$
\begin{aligned}
& r(p-1) \int_{M} \frac{|\nabla u|^{2}}{u} \Delta u+(r-1)(r(p-2)+1) \int_{M} \frac{|\nabla u|^{4}}{u^{2}} \\
= & C(p-2) \int_{M}|\nabla u|^{2}-\int_{M}(\Delta u)^{2},
\end{aligned}
$$

which is exactly (3.2).

Applying Theorem 4 for $q=0$ we get 


$$
\begin{aligned}
\rho \int_{M}|\nabla u|^{2} \leq & \frac{1}{2 m}((m-1) k+m) \int_{M}(\Delta u)^{2} \\
& -\frac{a(m+1) k}{m} \int_{M} \frac{|\nabla u|^{2}}{u} \Delta u \\
& +\left(a k+\frac{a^{2} k}{2 m}(m k+(m-1))\right) \int_{M} \frac{|\nabla u|^{4}}{u^{2}} .
\end{aligned}
$$

Note that (3.2) yields

$$
\begin{aligned}
-\frac{a(m+1) k}{m} \int_{M} \frac{|\nabla u|^{2}}{u} \Delta u= & -\frac{C(p-2)}{r(p-1)} \frac{a(m+1) k}{m} \int_{M}|\nabla u|^{2} \\
& +\frac{1}{r(p-1)} \frac{a(m+1) k}{m} \int_{M}(\Delta u)^{2} \\
& +\frac{(r-1)(r(p-2)+1)}{r(p-1)} \frac{a(m+1) k}{m} \int_{M} \frac{|\nabla u|^{4}}{u^{2}}
\end{aligned}
$$

Using this in (3.6) implies

$$
\begin{aligned}
& \left(\rho+\frac{C(p-2)}{r(p-1)} \frac{a(m+1) k}{m}\right) \int_{M}|\nabla u|^{2} \\
\leq & \left(\frac{1}{2 m}((m-1) k+m)+\frac{1}{r(p-1)} \frac{a(m+1) k}{m}\right) \int_{M}(\Delta u)^{2} \\
+ & \mathcal{F} \int_{M} \frac{|\nabla u|^{4}}{u^{2}},
\end{aligned}
$$

where

$$
\mathcal{F}=a k+\frac{a^{2} k}{2 m}(m k+(m-1))+\frac{(r-1)(r(p-2)+1)}{r(p-1)} \frac{a(m+1) k}{m} .
$$

We now set

$$
a=-\frac{(p-1) r(m+(m-1) k)}{2(m+1) k},
$$

so that

$$
\frac{1}{2 m}((m-1) k+m)+\frac{1}{r(p-1)} \frac{a(m+1) k}{m}=0 .
$$

Hence, (3.7) becomes

$$
\left(\rho-\frac{(p-2)(m+(m-1) k)}{2 m} C\right) \int_{M}|\nabla u|^{2} \leq \mathcal{F} \int_{M} \frac{|\nabla u|^{4}}{u^{2}} .
$$

By plugging (3.9) in (3.8) we get

$$
\mathcal{F}=\frac{m+(m-1) k}{8 m(m+1)^{2} k} \Theta(r),
$$

where

$$
\begin{aligned}
\Theta(r)= & -4 k m(m+1)(p-1) r \\
& +(p-1)^{2}(m+(m-1) k)(m k+(m-1)) r^{2} \\
& -4 k(m+1)^{2}(r-1)(r(p-2)+1)
\end{aligned}
$$


In conclusion, (3.10) implies that

$$
\begin{aligned}
& -\frac{m+(m-1) k}{8 m(m+1)^{2} k} \Theta(r) \int_{M} \frac{|\nabla u|^{4}}{u^{2}} \\
\leq & \left(\frac{(m+(m-1) k)(p-2)}{2 m} C-\rho\right) \int_{M}|\nabla u|^{2}
\end{aligned}
$$

where $\Theta(r)$ is given in (3.11).

We write

$$
\Theta(r)=c_{0} r^{2}+c_{1} r+c_{2}
$$

where

$$
\begin{aligned}
& c_{0}=(p-1)^{2}(m+(m-1) k)(m k+(m-1))-4 k(m+1)^{2}(p-2) \\
& c_{1}=-4 k(m+1)(2 m+3-p) \\
& c_{2}=4 k(m+1)^{2}
\end{aligned}
$$

We claim there exists $r \in \mathbb{R}$ so that $\Theta(r)=0$. Indeed, observe that

$$
\left(c_{1}\right)^{2}-4 c_{0} c_{2}=16 k(m+1)^{2} Q
$$

where

$$
\begin{aligned}
Q= & k(2 m+3-p)^{2}-(p-1)^{2}(m+(m-1) k)(m k+(m-1)) \\
& +4 k(m+1)^{2}(p-2) \\
= & m(m-1)(k+1)^{2}(p-1)\left(1+\frac{m+1}{m-1} \frac{4 k}{(k+1)^{2}}-p\right) \\
\geq & 0 .
\end{aligned}
$$

In the last line we used the hypothesis that

$$
p \leq 1+\frac{m+1}{m-1} \frac{4 k}{(k+1)^{2}}
$$

This proves that indeed there exists $r \in \mathbb{R}$ so that $\Theta(r)=0$. From (3.12) we get that

$$
C \geq \frac{2 m \rho}{(m+(m-1) k)(p-2)}
$$

which proves the lemma.

From [5] and Lemma [5] we obtain the following Sobolev inequality.

Proposition 6. Let $\left(M^{m}, g\right)$ be a compact Kähler manifold satisfying Ric $\geq \rho$ and $\operatorname{Vol}(M)=1$. For any $2<p \leq \frac{2 m}{m-1}$ we have the Sobolev inequality

$$
\left(\int_{M}|\phi|^{p}\right)^{\frac{2}{p}}-\int_{M} \phi^{2} \leq \frac{(m+(m-1) k)(p-2)}{2 m \rho} \int_{M}|\nabla \phi|^{2},
$$

for any $k>0$ such that

$$
p \leq 1+\frac{m+1}{m-1} \frac{4 k}{(k+1)^{2}}
$$


Proof. Consider the functional

$$
\mathcal{F}(\phi)=\frac{\left(\int_{M}|\phi|^{p}\right)^{\frac{2}{p}}-\int_{M} \phi^{2}}{\int_{M}|\nabla \phi|^{2}} .
$$

According to (3.1),

$$
\sup _{\phi \in C^{\infty}(M)} \mathcal{F}(\phi) \leq \frac{(n-1)(p-2)}{n \rho} .
$$

To improve (3.1), let $f$ be an extremum of the functional $\mathcal{F}$. By the argument in Ch. 6.8.2 of [2, we may assume that $f$ is positive, nonconstant and smooth. Denoting with

$$
\Lambda:=\sup _{\phi \in C^{\infty}(M)} \mathcal{F}(\phi),
$$

we may normalize $f$ so that it satisfies the partial differential equation

$$
\Delta f=\frac{1}{\Lambda}\left(f-f^{p-1}\right) .
$$

The result now follows from Lemma 5

Let us note that solving the equation

$$
p=1+\frac{m+1}{m-1} \frac{4 k}{(k+1)^{2}}
$$

in $k$ and plugging it in (3.15) implies that

$$
\left(\int_{M}|\phi|^{p}\right)^{\frac{2}{p}}-\int_{M} \phi^{2} \leq C_{S} \int_{M}|\nabla \phi|^{2},
$$

for any $\phi \in C^{\infty}(M)$, where

$$
C_{S}=\frac{p-2}{(p-1) 2 m \rho}(2 m+p+1-2 \sqrt{(m+1)(2 m-(m-1) p)}) .
$$

This proves the first part of Theorem 2

As another consequence of Lemma [5. we obtain the following Beckner type inequality, that completes the proof of Theorem 2 .

Proposition 7. Let $\left(M^{m}, g\right)$ be a Kähler manifold of complex dimension $m \geq 2$ and with Ricci curvature $\operatorname{Ric} \geq \rho$ and $\operatorname{Vol}(M)=1$. For any $1<p \leq 2$ we have the inequality

$$
\int_{M} \phi^{2}-\left(\int_{M} \phi^{\frac{2}{p}}\right)^{p} \leq C_{B} \int_{M}|\nabla \phi|^{2}
$$

where

$$
C_{B}=\frac{p-1}{p} \frac{2 m}{((m-1) p+2) \rho} .
$$

Proof. We follow the proof in $[8$. For a fixed $\phi$, let $f(t)$ be the solution of

$$
\begin{aligned}
f_{t} & =\Delta f \\
f(0) & =\phi^{\frac{2}{p}} .
\end{aligned}
$$

Define

$$
\Lambda(t)=\int_{M} f^{p}-\left(\int_{M} f\right)^{p} .
$$


Our goal is to establish a differential inequality for $\Lambda(t)$, for all $t>0$. For this, it is convenient to denote

$$
\begin{aligned}
q & =\frac{2-p}{p-1} \\
u & =f^{p-1} .
\end{aligned}
$$

Clearly, $q \geq 0$ and $u>0$. We have the following identities (cf. [8])

$$
\frac{p-1}{p} \Lambda^{\prime}(t)=-\int_{M}|\nabla u|^{2} u^{q}
$$

and

$$
\begin{aligned}
\frac{p-1}{p} \Lambda^{\prime \prime}(t) \geq & 2 \int_{M}\left|\nabla^{2} u\right|^{2} u^{q}+2 \rho \int_{M}|\nabla u|^{2} u^{q} \\
& +q \int_{M}|\nabla u|^{4} u^{q-2} .
\end{aligned}
$$

Indeed, we have

$$
\begin{aligned}
\Lambda^{\prime}(t) & =p \int_{M}\left(f_{t}\right) f^{p-1}-p\left(\int_{M} f\right)^{p-1} \int_{M} f_{t} \\
& =p \int_{M}(\Delta f) f^{p-1} \\
& =-p(p-1) \int_{M}|\nabla f|^{2} f^{p-2} .
\end{aligned}
$$

Using that $f=u^{\frac{1}{p-1}}$, this immediately implies (3.20). Note that $u$ verifies the equation

$$
u_{t}=\Delta u+q|\nabla u|^{2} u^{-1} \text {. }
$$

Therefore,

$$
\frac{d}{d t}|\nabla u|^{2}=2\langle\nabla u, \nabla \Delta u\rangle+2 q\left\langle\nabla u, \nabla|\nabla u|^{2}\right\rangle u^{-1}-2 q|\nabla u|^{4} u^{-2} .
$$

The Bochner formula yields

$$
\Delta|\nabla u|^{2} \geq 2\left|\nabla^{2} u\right|^{2}+2 \rho|\nabla u|^{2}+2\langle\nabla \Delta u, \nabla u\rangle .
$$

Combining with (3.22) we have

$$
\begin{aligned}
\frac{d}{d t}|\nabla u|^{2} \leq & \Delta|\nabla u|^{2}-2\left|\nabla^{2} u\right|^{2}-2 \rho|\nabla u|^{2} \\
& +2 q\left\langle\nabla u, \nabla|\nabla u|^{2}\right\rangle u^{-1}-2 q|\nabla u|^{4} u^{-2} .
\end{aligned}
$$

Now take a derivative in $t$ of (3.20) and integrate by parts to get

$$
\begin{aligned}
\frac{p-1}{p} \Lambda^{\prime \prime}(t) & =-\int_{M} \frac{d}{d t}\left(|\nabla u|^{2}\right) u^{q}-q \int_{M}|\nabla u|^{2}\left(\Delta u+q|\nabla u|^{2} u^{-1}\right) u^{q-1} \\
& \geq 2 \int_{M}\left|\nabla^{2} u\right|^{2} u^{q}+2 \rho \int_{M}|\nabla u|^{2} u^{q}+q \int_{M}|\nabla u|^{4} u^{q-2}
\end{aligned}
$$

which proves 3.21). 
According to Lemma 5 we have for any $0 \leq k \leq \frac{m}{m-1}$,

$$
\begin{aligned}
(m+(m-1) k) \int_{M}\left|\nabla^{2} u\right|^{2} u^{q} \geq & (m-(m-1) k) \rho \int_{M}|\nabla u|^{2} u^{q}+A_{2} \int_{M}|\nabla u|^{2}(\Delta u) u^{q-1} \\
& -B_{2} \int_{M}|\nabla u|^{4} u^{q-2},
\end{aligned}
$$

where

$$
\begin{aligned}
A_{2}= & 2(m+1) a k+\frac{1}{2}(1-k) m q-\frac{3}{2} k q \\
B_{2}= & ((m-1)+m k) k a^{2}+(2-(k+1) q) m k a \\
& +\frac{m}{4}(1-k)^{2} q^{2}+\frac{q(q-1)}{2}(m(k-1)+k) .
\end{aligned}
$$

Let

$$
a=\frac{3 q}{4(m+1)}-\frac{m}{4(m+1)} \frac{1-k}{k} q,
$$

for which $A_{2}=0$. This yields

$$
\begin{aligned}
\int_{M}\left|\nabla^{2} u\right|^{2} u^{q} \geq & \frac{m-(m-1) k}{m+(m-1) k} \rho \int_{M}|\nabla u|^{2} u^{q} \\
& -\frac{B_{2}}{m+(m-1) k} \int_{M}|\nabla u|^{4} u^{q-2},
\end{aligned}
$$

for $B_{2}$ specified in (3.23). It is more convenient to denote

$$
2 \sigma=\frac{1-k}{k} q,
$$

where we are assuming $\sigma>0$, so in particular $0 \leq k \leq \frac{m}{m-1}$. Then (3.24) becomes

$$
a=\frac{3 q}{4(m+1)}-\frac{m}{2(m+1)} \sigma
$$

and (3.25) yields

$$
\begin{aligned}
\int_{M}\left|\nabla^{2} u\right|^{2} u^{q} \geq & \frac{q+2 m \sigma}{(2 m-1) q+2 m \sigma} \rho \int_{M}|\nabla u|^{2} u^{q} \\
& -\frac{1}{\left(1+\frac{2}{q} \sigma\right)\left(2 m-1+\frac{2 m}{q} \sigma\right)} B_{3} \int_{M}|\nabla u|^{4} u^{q-2}
\end{aligned}
$$

where

$$
\begin{aligned}
B_{3}= & \left((2 m-1)+\frac{2(m-1)}{q} \sigma\right) a^{2}+2\left((1-q)+\left(\frac{2}{q}-1\right) \sigma\right) m a \\
& +m \sigma^{2}+\frac{q-1}{2}\left(q-2(m-1) \sigma-\frac{4}{q} m \sigma^{2}\right) .
\end{aligned}
$$


Using (3.27) we obtain

$$
\begin{aligned}
B_{3}= & \frac{(2 m-1) q}{16(m+1)^{2}}(8(m+1)-(8 m-1) q) \\
& +\frac{3 m-1}{8(m+1)^{2}}(8(m+1)-(8 m-1) q) \sigma \\
& +\frac{m}{4(m+1)^{2}}\left(\left(2 m^{2}-11 m+2\right)+\frac{8(m+1)}{q}\right) \sigma^{2} \\
& +\frac{m^{2}(m-1)}{2(m+1)^{2} q} \sigma^{3} .
\end{aligned}
$$

Plugging (3.28) into (3.21) yields

$$
\begin{aligned}
\frac{p-1}{p} \Lambda^{\prime \prime}(t) \geq & \left(\frac{q+2 m \sigma}{(2 m-1) q+2 m \sigma}+1\right) 2 \rho \int_{M}|\nabla u|^{2} u^{q} \\
& +\Upsilon \int_{M}|\nabla u|^{4} u^{q-2}
\end{aligned}
$$

where

$$
\Upsilon=q-\frac{2 B_{3}}{\left(1+\frac{2}{q} \sigma\right)\left(2 m-1+\frac{2 m}{q} \sigma\right)} .
$$

It can be checked directly that $\Upsilon \geq 0$ is equivalent to $\mathcal{E} \geq 0$, where

$$
\begin{aligned}
\mathcal{E}= & (2 m-1) q(8 m(m+1)+(8 m-1) q) \\
& +2(3 m-1)(8 m(m+1)+(8 m-1) q) \sigma \\
& +4 m\left(8 m(m+1) \frac{1}{q}-\left(2 m^{2}-11 m+2\right)\right) \sigma^{2} \\
& -8 m^{2}(m-1) \frac{1}{q} \sigma^{3} .
\end{aligned}
$$

It is easy to see that $\mathcal{E} \geq 0$ for $\sigma=1+\frac{q}{2 m}$, which by (3.29) proves

$$
\frac{p-1}{p} \Lambda^{\prime \prime}(t) \geq \frac{(m+1) q+2 m}{m(q+1)} 2 \rho \int_{M}|\nabla u|^{2} u^{q} .
$$

Together with (3.20) this yields

$$
\begin{aligned}
\Lambda^{\prime \prime}(t) & \geq \frac{(m+1) q+2 m}{m(q+1)} 2 \rho\left(-\Lambda^{\prime}(t)\right) \\
& =\frac{(m-1) p+2}{m} 2 \rho\left(-\Lambda^{\prime}(t)\right) .
\end{aligned}
$$

Integrating first from $t=0$ to $t=s$ and then from $s=0$ to $s=\infty$ implies

$$
\begin{aligned}
\int_{M} \phi^{2}-\left(\int_{M} \phi^{\frac{2}{p}}\right)^{p} & \leq\left(-\Lambda^{\prime}(0)\right) \frac{m}{(m-1) p+2} \frac{1}{2 \rho} \\
& =\frac{p-1}{p} \frac{m}{(m-1) p+2} \frac{2}{\rho} \int_{M}|\nabla \phi|^{2} .
\end{aligned}
$$

This proves the result. 


\section{Diameter estimate For KähleR MAnifolds}

In this section, we use the inequalities obtained in the previous sections to prove Theorem 3 .

Theorem 8. Let $\left(M^{m}, g\right)$ be a Kähler manifold of complex dimension $m \geq 2$ and with Ricci curvature Ric $\geq \rho$. Then

$$
\operatorname{diam}(M) \leq \frac{\pi}{\sqrt{\rho}} \sqrt{2 m-1}\left(1-\frac{1}{24 m}\right)
$$

Proof. According to [3], if the Sobolev inequality

$$
\left(\int_{M}|\phi|^{p}\right)^{\frac{2}{p}}-\int_{M} \phi^{2} \leq A \int_{M}|\nabla \phi|^{2}
$$

holds for some $p>2$, then

$$
\operatorname{diam}(M) \leq \pi \frac{\sqrt{2 p A}}{p-2} .
$$

In our setting, applying Proposition 6 for

$$
p=1+\frac{m+1}{m-1} \frac{4 k}{(k+1)^{2}},
$$

we get

$$
\operatorname{diam}(M) \leq \frac{\pi}{\sqrt{\rho}} \sqrt{\frac{p(m+(m-1) k)}{m(p-2)}} .
$$

Recall that the Bonnet-Myers estimate is

$$
\operatorname{diam}(M) \leq \frac{\pi}{\sqrt{\rho}} \sqrt{2 m-1} .
$$

To show that (4.2) improves this estimate, we compute

$$
\begin{aligned}
& \sqrt{2 m-1}-\sqrt{\frac{p(m+(m-1) k)}{m(p-2)}} \\
= & \frac{1}{m(p-2)}\left(\sqrt{2 m-1}+\sqrt{\frac{p(m+(m-1) k)}{m(p-2)}}\right)^{-1} \mathcal{S},
\end{aligned}
$$

where

$$
\mathcal{S}=m(2 m-1)(p-2)-p(m+(m-1) k) .
$$

Using $p$ from (4.1) we get

$$
\begin{aligned}
\mathcal{S}= & m(2 m-1)\left(\frac{m+1}{m-1} \frac{4 k}{(k+1)^{2}}-1\right) \\
& -(m+(m-1) k)\left(\frac{m+1}{m-1} \frac{4 k}{(k+1)^{2}}+1\right) \\
= & \frac{1}{(m-1)(k+1)^{2}} \Omega
\end{aligned}
$$


where

$$
\begin{aligned}
\Omega= & 4 m(2 m-1)(m+1) k-m(2 m-1)(m-1)(k+1)^{2} \\
& -4(m+(m-1) k)(m+1) k-(m+(m-1) k)(m-1)(k+1)^{2} \\
= & 4(m+1)(m-1)(2 m-k) k-(m-1)\left(2 m^{2}+(m-1) k\right)(k+1)^{2} .
\end{aligned}
$$

Hence, using this in (4.4) we get

$$
\mathcal{S}=\frac{1}{(k+1)^{2}} \Psi
$$

where

$$
\begin{aligned}
\Psi & =4(m+1)(2 m-k) k-\left(2 m^{2}+(m-1) k\right)(k+1)^{2} \\
& =(1-k)\left((m-1)(1-k)^{2}-\left(2 m^{2}+9 m-1\right)(1-k)+8 m\right) .
\end{aligned}
$$

We now set

$$
k=1-\frac{1}{2 m}
$$

for which we note that

$$
\begin{aligned}
p-2 & =\frac{1}{(m-1)(k+1)^{2}}\left(8-8(1-k)-(m-1)(1-k)^{2}\right) \\
& >0 .
\end{aligned}
$$

Furthermore, it is easy to see that $\Psi \geq 2$. Hence, by (4.5), this implies

$$
\mathcal{S} \geq \frac{2}{(k+1)^{2}}
$$

By (4.3) we get

$$
\begin{aligned}
& \sqrt{2 m-1}-\sqrt{\frac{p(m+(m-1) k)}{m(p-2)}} \\
= & \frac{2}{m(p-2)(k+1)^{2}}\left(\sqrt{2 m-1}+\sqrt{\frac{p(m+(m-1) k)}{m(p-2)}}\right)^{-1} .
\end{aligned}
$$

From (4.7) and (4.6) we have

$$
\begin{aligned}
m(p-2)(k+1)^{2} & =\frac{m}{m-1}\left(8-\frac{4}{m}-\frac{m-1}{4 m^{2}}\right) \\
& \leq \frac{8 m}{m-1} .
\end{aligned}
$$

Furthermore, note that

$$
p(m+(m-1) k) \leq \frac{2 m}{m-1}(2 m-1) .
$$

Hence, (4.9) yields

$$
\sqrt{2 m-1}-\sqrt{\frac{p(m+(m-1) k)}{m(p-2)}} \geq \frac{m-1}{8 m \sqrt{2 m-1}} .
$$


It follows that

$$
\sqrt{2 m-1}-\sqrt{\frac{p(m+(m-1) k)}{m(p-2)}} \geq \frac{\sqrt{2 m-1}}{24 m}
$$

for all $m \geq 2$.

By (4.2) and (4.10) we obtain

$$
\operatorname{diam}(M) \leq \frac{\pi}{\sqrt{\rho}} \sqrt{2 m-1}\left(1-\frac{1}{24 m}\right) .
$$

This proves the theorem.

We conclude with a different approach to the diameter estimate, which we learned from Jiaping Wang.

Proposition 9. Let $\left(M^{m}, g\right)$ be a Kähler manifold of complex dimension $m \geq 2$ and with Ricci curvature Ric $\geq 2 m-1$. Let $d=\operatorname{diam}(M)$ be the diameter of $M$. Then

$$
2(2 m-1) \leq\left(\frac{\pi}{d}\right)^{2} \frac{\int_{0}^{\frac{d}{2}} \sin ^{2}\left(\frac{\pi r}{d}\right) \sin ^{2 m-1} r d r}{\int_{0}^{\frac{d}{2}} \cos ^{2}\left(\frac{\pi r}{d}\right) \sin ^{2 m-1} r d r}
$$

Proof. Recall that on a Kähler manifold with Ric $\geq 2 m-1$ the first nonzero eigenvalue of the Laplacian satisfies

$$
\lambda_{1}(M) \geq 2(2 m-1) .
$$

Indeed, this also follows by setting $p=2$ in Proposition 7 .

Let $p, q \in M$ be so that $d=d(p, q)$. Then we have the following well known estimate

$$
\lambda_{1}(M) \leq \max \left\{\mu_{1}\left(B\left(p, \frac{d}{2}\right), \mu_{1}\left(B\left(q, \frac{d}{2}\right)\right)\right)\right\},
$$

where $\mu_{i}(\Omega)$ denotes the first Dirichlet eigenvalue of $\Omega$. By using Cheng's comparison theorem [6], we know that

$$
\max \left\{\mu_{1}\left(B\left(p, \frac{d}{2}\right), \mu_{1}\left(B\left(q, \frac{d}{2}\right)\right)\right)\right\} \leq \mu_{1}\left(\widetilde{B}\left(\frac{d}{2}\right)\right),
$$

where $\mu_{1}\left(\widetilde{B}\left(\frac{d}{2}\right)\right)$ denotes the first Dirichlet eigenvalue of the ball of radius $\frac{d}{2}$ in the sphere $\left(\mathbb{S}^{2 m}, \widetilde{g}\right)$ normalized so that $\operatorname{Ric}_{\widetilde{g}}=2 m-1$. Hence, (4.12) and (4.11) imply that

$$
2(2 m-1) \leq \mu_{1}\left(\widetilde{B}\left(\frac{d}{2}\right)\right) .
$$

To estimate the right side of (4.13) we use the variational characterization

$$
\bar{\mu}_{1}\left(\widetilde{B}\left(\frac{d}{2}\right)\right) \leq \frac{\int_{\widetilde{B}\left(\frac{d}{2}\right)}|\nabla \phi|_{\widetilde{g}}^{2}}{\int_{\widetilde{B}\left(\frac{d}{2}\right)} \phi^{2}},
$$

for any function $\phi$ supported in $\widetilde{B}\left(\frac{d}{2}\right)$. Choosing a rotationally symmetric function

$$
\phi(r)=\cos \left(\frac{r \pi}{d}\right)
$$


we get by (4.14) that

$$
\bar{\mu}_{1}\left(\widetilde{B}\left(\frac{d}{2}\right)\right) \leq\left(\frac{\pi}{d}\right)^{2} \frac{\int_{0}^{\frac{d}{2}} \sin ^{2}\left(\frac{\pi r}{d}\right) \sin ^{2 m-1} r d r}{\int_{0}^{\frac{d}{2}} \cos ^{2}\left(\frac{\pi r}{d}\right) \sin ^{2 m-1} r d r} .
$$

Using (4.13), this proves the proposition.

We use Proposition 9 to prove the following result.

Theorem 10. Let $\left(M^{m}, g\right)$ be a Kähler manifold of complex dimension $m \geq 2$ and with Ricci curvature Ric $\geq 2 m-1$. Then

$$
\operatorname{diam}(M) \leq \pi\left(1-\frac{1}{200 \sqrt{m} \ln m}\right)
$$

Proof. Let

$$
\varepsilon=\frac{\pi}{d}-1>0
$$

and assume by contradiction that

$$
\varepsilon<\frac{1}{100 \sqrt{m} \ln m} .
$$

We have the inequalities

$$
\begin{aligned}
1 & \geq \cos (\varepsilon t) \geq 1-\frac{1}{2} \varepsilon^{2} t^{2} \\
\varepsilon t & \geq \sin (\varepsilon t) \geq \frac{1}{2} \varepsilon t,
\end{aligned}
$$

for any $0<t<\frac{\pi}{2}$. Denote with

$$
I_{n}=\int_{0}^{\frac{d}{2}} \sin ^{n} r d r
$$

Integrating by parts we get the relation

$$
I_{2 k+1}=\frac{2 k}{2 k+1} I_{2 k-1}-\frac{1}{2 k+1} \sin ^{2 k}\left(\frac{d}{2}\right) \cos \left(\frac{d}{2}\right),
$$

for any $k \geq 1$. As

$$
\begin{aligned}
\cos \left(\frac{d}{2}\right) & =\cos \left(\frac{\pi}{2}-\frac{\varepsilon}{1+\varepsilon} \frac{\pi}{2}\right) \\
& =\sin \left(\frac{\varepsilon}{1+\varepsilon} \frac{\pi}{2}\right),
\end{aligned}
$$

we get from (4.16) and (4.17) that

$$
\frac{1}{2} \varepsilon \leq \cos \left(\frac{d}{2}\right) \leq 2 \varepsilon .
$$

This implies that

$$
I_{2 k+1} \geq \frac{2 k}{2 k+1} I_{2 k-1}-\frac{2 \varepsilon}{2 k+1} .
$$

Iterating, we get

$$
I_{2 m+1} \geq \frac{2^{2 m}(m !)^{2}}{(2 m+1) !}\left(1-\cos \frac{d}{2}\right)-2 \varepsilon\left(\frac{1}{2 m+1}+\frac{1}{2 m-1}+\ldots+\frac{1}{3}\right) .
$$


Using Stirling inequalities

$$
\sqrt{2 \pi}\left(\frac{n}{e}\right)^{n} \sqrt{n} \leq n ! \leq e\left(\frac{n}{e}\right)^{n} \sqrt{n},
$$

together with (4.16) and (4.19) it follows that

$$
I_{2 m+1} \geq \frac{1}{\sqrt{2 m+1}}-2 \varepsilon \ln (2 m+1) .
$$

In particular, 4.16) and (4.21) imply that

$$
I_{2 m+1} \geq \frac{4}{5} \frac{1}{\sqrt{2 m+1}}
$$

Note that

$$
\frac{\int_{0}^{\frac{d}{2}} \sin ^{2}\left(\frac{\pi r}{d}\right) \sin ^{2 m-1} r d r}{\int_{0}^{\frac{d}{2}} \cos ^{2}\left(\frac{\pi r}{d}\right) \sin ^{2 m-1} r d r}=\frac{I_{2 m-1}}{\int_{0}^{\frac{d}{2}} \cos ^{2}\left(\frac{\pi r}{d}\right) \sin ^{2 m-1} r d r}-1 .
$$

From (4.17) we get

$$
\begin{aligned}
\cos ^{2}\left(\frac{\pi r}{d}\right) & =(\cos (\varepsilon r) \cos r-\sin (\varepsilon r) \sin r)^{2} \\
& \geq \cos ^{2}(\varepsilon r) \cos ^{2} r-2 \sin (\varepsilon r) \cos (\varepsilon r) \sin r \cos r \\
& \geq\left(1-\varepsilon^{2} r^{2}\right) \cos ^{2} r-2 \varepsilon r \sin r \cos r \\
& \geq \cos ^{2} r-4 \varepsilon \cos r .
\end{aligned}
$$

Then it follows that

$$
\begin{aligned}
\int_{0}^{\frac{d}{2}} \cos ^{2}\left(\frac{\pi r}{d}\right) \sin ^{2 m-1} r d r & \geq \int_{0}^{\frac{d}{2}} \cos ^{2} r \sin ^{2 m-1} r d r-4 \varepsilon \int_{0}^{\frac{d}{2}} \cos r \sin ^{2 m-1} r d r \\
& =I_{2 m-1}-I_{2 m+1}-\frac{2 \varepsilon}{m} \sin ^{2 m}\left(\frac{d}{2}\right) \\
& \geq I_{2 m-1}-I_{2 m+1}-\frac{2 \varepsilon}{m} .
\end{aligned}
$$

From (4.18), we deduce that

$$
\int_{0}^{\frac{d}{2}} \cos ^{2}\left(\frac{\pi r}{d}\right) \sin ^{2 m-1} r d r \geq \frac{1}{2 m+1} I_{2 m-1}-\frac{2 \varepsilon}{m} .
$$

However, by (4.16) and (4.22) we get

Therefore, this implies

$$
\frac{2 \varepsilon}{m}<\frac{1}{3} \frac{1}{2 m+1} I_{2 m+1}
$$

$$
\int_{0}^{\frac{d}{2}} \cos ^{2}\left(\frac{\pi r}{d}\right) \sin ^{2 m-1} r d r \geq \frac{2}{3} \frac{1}{2 m+1} I_{2 m-1} .
$$

Hence, by (4.23) and (4.24) we infer that

$$
\frac{\int_{0}^{\frac{d}{2}} \sin ^{2}\left(\frac{\pi r}{d}\right) \sin ^{2 m-1} r d r}{\int_{0}^{\frac{d}{2}} \cos ^{2}\left(\frac{\pi r}{d}\right) \sin ^{2 m-1} r d r} \leq \frac{3}{2}(2 m+1) .
$$

By Proposition 9 we get

$$
2(2 m-1) \leq \frac{3}{2}(1+\varepsilon)^{2}(2 m+1),
$$


which contradicts (4.16).

Therefore, we have

which proves the result.

$$
\frac{\pi}{d}-1 \geq \frac{1}{100 \sqrt{m} \ln m},
$$

\section{REFERENCES}

[1] D. Bakry, L'hypercontractivite et son utilisation en theorie des semigroupes" in Ecole d'Ete de Probabilites de Saint-Flour, 1992, Lecture Notes in Math. 1581, Springer-Verlag, Berlin, 1994, 1-114.

[2] D. Bakry, I. Gentil and M. Ledoux, Analysis and geometry of Markov diffusion operators, A Series of Comprehensive Studies in Mathematics, Springer 348 (2013).

[3] D. Bakry and M. Ledoux, Sobolev inequalities and Myers's diameter theorem for an abstract Markov generator, Duke Math. J. 85, Number 1 (1996), 253-270.

[4] F. Baudoin, Geometric Inequalities on Riemannian and sub-Riemannian manifolds by heat semigroups techniques, arXiv:1801.05702.

[5] M.-F. Bidaut-Veron and L. Veron, Nonlinear elliptic equations on compact Riemannian manifolds and asymptotics of Emden equations, Invent. Math., 106 (1991), 489-539.

[6] S.Y. Cheng, Eigenvalue comparison theorems and its geometric application, Math. Z. 143 (1975), 289-297.

[7] J. Dolbeault, M. J. Esteban, M. Kowalczyk, and M. Loss, Sharp Interpolation Inequalities on the Sphere: New Methods and Consequences, Chinese Annals of Mathematics, Series B, 2013, Volume 34, Issue 1, pp 99-112

[8] I. Gentil and A. Zugmeyer, A family of Beckner inequalities under various curvaturedimension conditions, arXiv:1903.00214

[9] B. Gidas and J. Spruck, Global and local behavior of positive solutions of nonlinear elliptic equations, Commun. Pure Appl. Math. (1981), 525-598.

[10] E. Hebey, Nonlinear Analysis on Manifolds: Sobolev Spaces and Inequalities, Courant Institute of Mathematical Sciences, Lecture Notes in Mathematics, 5, 1999.

[11] E. Hebey and M. Vaugon, Meilleures constantes dans le theoreme d'inclusion de Sobolev, Annales de l'I. H. P. (1996), no 1, 57-93.

[12] M. Ledoux, The geometry of Markov diffusion generators. Probability theory. Ann. Fac. Sci. Toulouse Math. (6) 9 (2000), no. 2, 305-366.

[13] P. Li and J. Wang, Comparison theorem for Kähler manifolds and positivity of spectrum, J. Differential Geom. 69 (2005), 43-74.

[14] G. Liu, Kähler manifolds with Ricci curvature lower bound, Asian J. Math. 18 (2014), no. 1, 69-99.

[15] G. Liu and Y. Yuan, Diameter rigidity for Kähler manifolds with positive bisectional curvature, to appear in Math Z.

[16] M. Obata, The conjectures on conformal transformations of Riemannian manifolds, J.Differential Geometry 6 (1971/72), 247-258.

[17] L. Saloff-Coste, Precise estimates on the rate at which certain diffusions tend to equilibrium, Math. Z. 217, 641-677 (1994)

[18] Y. Tsukamoto, On Kählerian manifolds with positive holomorphic sectional curvature, Proc. Japan Acad. 33 (1957), 333-335.

[19] L. F. Tam and C. Yu, Some comparison theorems for Kähler manifolds, Manuscripta Math. 137 (2012), no. 3-4, 483-495.

E-mail address: fabrice.baudoin@uconn.edu

Department of Mathematics, University of Connecticut, Storrs, CT 06268, USA

E-mail address: ovidiu.munteanu@uconn.edu

Department of Mathematics, University of Connecticut, Storrs, CT 06268, USA 